\title{
Sachregister zu Band 75
}

Die fett gedruckten Zahlen bezeichnen Eigenarbeiten. Bb. = Buchbesprechung.

A

Ablatio retinae 102, 382

- $\quad$ eine ungewöhnliche Form der -326

- und Migräne 191

- $\quad$ anatomisch-klinische Studie einesFalles von operierter - 392

- $\quad$ Komplikation bei einem nachGonin operierten Fall von - 389

Akkommodation, subnormale 384 Albinismus bei einem Mischling 384 Aleukia haemorrhagica, Augen-

veränderungen bei - 122 Alpha-Krystallin, Organspezifi-tät undAntigencharakter des-378

Amblyopie ohne Spiegelbefund 354

- Bleivergiftung oder Magenkrebsals Ursache einer toxischen - 108

Angiopathia retinae traumatica

ad maculam 118 Angio-Retikulo-Xanthom der

Netzhaut 399 Angioidstreifen und ihre Bezie-

hung zu zentraler Chorioiditis 200 Aphakia congenita 386 Arachnodaktylie mit totaler Lin-

senluxation 388 Artefizielle Augenentzündun-

gen, objektiver Nachweis der -

109 Arteria hyaloidea persistens,

Anatomie der - 168

- $\quad$ Reste einer - in einem CanalisCloqueti 197

Arteriosklerose, Messungen an

den Netzhautarterien bei begin-

nender - 381 Atlas, stereoskopischer - der äuße-

ren Erkrankungen des Auges 205

(Bb.) Auge, Entschädigung bei Verlust

eines - 126 Augenentzündungen, objektiver

Nachweis artefizieller - 109 Augenkrankheiten, vom Stand-

punkt der Eugenik aus betrachtet

39o Augenpol, scheibenförmige Degeneration am hinteren - 216 Augenspiegelung, gleichzeitige $-$

beider Augen 191 Augenverletzungen, Differential-diagnose zwischen Augenerkran-kungen und -205 (Bb.)

Autoserumtherapie gegen Augen-

tuberkulose 118 Avertin 373

B

Basedowoperation, progredierter

Exophthalmus nach - 137 Bildbetrachtung, Prävalenz des 
einen Auges in der - 394 Bindehaut s. Conjunctiva Bleivergiftung oder Magenkrebs als Ursache einer toxischen Amblyopie 108 Blepharoconj unctivitis 198 Blepharophimosis medialis se-nilis und Lockerung des Liga-mentum canthi interni 118 Blindenwesen 204, 283, 401 Blindheit und Stauungspapille bei

Guernsey-Stierkälbern 383 Brill en aus splitterfreiem Glas 105 Brillengestell, Trägerrahmen für - 100

B u 1 b u s, Veränderungen am - durch Druck von Orbitaltumoren 264

Bulbuspuls, plethysmographische Untersuchungen über den -317

Bulbusverknöcherung, Röntgen-befund bei - no

C Canalis Cloqueti mit Resten einer

Arteria hyaloidea persistens 197 Chalazionpinzette, Heftpflaster-

streifen zum Fixieren der - 97 Chorioidea, progressive Atrophie

der - mit Pigmentdegeneration

der Netzhaut 279

- $\quad$ Schaffung flächenhafter Verlö-tungen der Retina mit der - durch Endothermie 207

Chorioiditis, Beziehung der zen-tralen - zu Angioidstreifen 200

- guttata no

Conj unctivitis, Grenzstrahlenbe-handlung der C. acuta und chro-nica catarrhalis 285

- Beziehungen der gonorrhoischen

- $\quad$ zur Genitalgonorrhoe 396

- $\quad$ knötchenförmige - durch Fett-imprägnation 369

Cornea, muzinöse Degeneration der

- $\quad$ 190Corneatransplantation 194

Sachregister

zu Band 75.

403

$\mathrm{D}$

Dakryozystitis, pathologische

Anatomie der Operation bei -

120 Degeneration, scheibenförmige -

am hinteren Augenpol 216 Denigsche Operation gegen Pan-

nus trachomatosus 194 Deviationskrämpf e 104 Diabetes, traumatischer nach Ver-

letzungen des Zentralnerven-

systems 128 Divergenzzittern 191 DruckentlastendeOperationen,

Einfluß vielfacher - bei Hydr-

ophthalmus congenitus und gleich-

zeitiger Entstehung von hoher

Myopie 17 Drucksteigerung, intrakranielle

- mit Stauungspapille 385 Dynamometer, Baurmannsches

385

$\mathrm{E}$

Endothermie, Schaffung flächen-

hafter Verlötungen der Retina

mit der Chorioidea durch - 207

Enophthalmus tra umaticus 186 
Enzephalitis nach Masern 384

- $\quad$ Retitinitis pseudoalbuminurica(stellata) nach - no

Entropium spasticum, Heft-pflaster-Schienenverband bei - 96

Enukleation, Technik der - 96

Epithelzyste im Glaskörper 98

Ericiskop von Peter 101

Eugenik, Augenkrankheiten vom Standpunkt der E. aus betrachtet

39o Euphakin 116 Exenteratio orbito-sinualis

nach Golowin 196 Exophthalmus bei benigner Or-

bitalgeschwulst 197

bei Säuglingsskorbut 373

pulsierender, entstanden durch erne Revolverkugel 190

progredienter - nach Basedow-operation 137

$\Gamma$

Farbenanalyse, der pseudoiso-

chromatischen Tafeln 196 Fettimprägnation, knötchenför-

mige Conjunctivitis durch - 369 Feuerlamelle, histologischer Be-

fund der F. der Linsenvorder-

kapsel 387 Fixationsvorrichtung für Peri-

metrie 105

Foramen opticum, Röntgenologie

des - 27 Frühj ahrskatarrh, Ätiologie und

Therapie des -119

(i

Gefäßsystem, peripheres - und

Auge 185 Gehirntumoren, diagnostische Be-

deutung der Kopfhaltung für die

Erkennung von - 400 Glaskörper, Epithelzyste im - 98 Glaskörperabschnitt, der vordere

- $\quad$ im gesunden und kranken Auge

379

Glassplitter, gezackter - am Bo-den der vorderen Kammer 191

Glaukom, Resultate der Zyklodi-alyse bei - 375

Glioma retinae, doppelseitiges 187

Goninsche Operation 102, 196, 389, 392

Gonorrhoe, Beziehungen der Conjunctiva! zur Genital- - 396

Grenzstrahlen, Tiefenwirkung der

- $\quad$ am Auge 32

Wirkung der - auf augenpatho-gene Keime 280

Behandlung der Conjunctivitis acuta und chronica catarrhalis mit -285

$\mathrm{H}$

Hämangioblastom des Kleinhirns

400 Hämangiom des Augenlids 117 Heftpflaster-Schienverband

bei Entropium spasticum 96 Hemianästhesie, Keratitis neuro-

paralytica bei einem Syndrom

der alternierenden - 124 Heniianopsie, topische Diagnostik

der -105 
- $\quad$ Schwangerschaftsveränderung derHypophyse als Ursache bitempo-raler - 343

Herpes febrilis des Lidrandes und

der Bindehaut 99 Hippelsche Krankheit 399 Hornhaut s. Cornea Hydrophthalmus congenitus,

Einfluß vielfacher druckentlasten-

der Operationen bei - und gleich-

zeitige Entstehung von hoher

Myopie 17 Hypernephrom, metastatisches -

des Auges 326 Hypophyse, Beziehungen zwischen

Thyreoidea und - 137

- $\quad$ Schwangerschaftsveränderung der-als Ursache bitemporaler Heniianopsie 343

404

Sachregister zu Band 75.

I Idiotie, familiäre amaurotische 198,

199, 386 Ignipunktur Ill Iris, Sarkom der - 257

, 1

Jagdverletzungen 190 Jodbehandlung der Katarakta se-nilis 129

$\mathrm{K}$

Kalkablagerungen im Plexus cho-rioideus bei Retinitis pigmentosa 164

Kammerwasser, Zirkulation des

- $\quad$ 377Kapillarsystem, Bau, Tonus und

Durchlässigkeit des - 185 Katarakt bei postoperativer Te-tanie 374

künstliche Schnellreifung der - 371

Resorption der K. senilis 198

- Jodbehandlung der K. senilis 129Kataraktoperation 119

Altersstarextraktion in der Kap-sel 1

eine erfolgreiche - durch Kuh-hornstoß 55

eine nach intrakapsulärer Ex-traktion zurückbleibende Mem-bran zwischen Glaskörper und

Vorderkammer 378

Vergleich der Resultate der Al-tersstaroperation mit und ohne vorbereitetem Bindehautlappen 95, 166

das histologische Bild des vor-deren Augenabschnittes unmittel-bar nach intrakapsulärer Extrak-

tion 379

Kayser-Fleischerscher Ringbei Wilsonscher Krankheit 103

Keilbeinhöhle, Neuritis optica verursacht durch Erkrankung der

$-\quad 383$

Keratitis interstitialis profun-

da 187 Keratitis neuroparalytica bei

einem Syndrom der alternieren-

den Hemiästhesie 124 Keratokonus 187

- $\quad$ operative Therapie der $-387 \mathrm{~K} 1$ e i n h i r n , Hämangioblastom des-

400 Kopfhaltung, diagnostische Be-

deutung der - für die Erkennung

von Gehirntumoren 400 Korneosklerale Wunde, geheilte

2 ccm lange -115

Krystallin, Organspezifität und 
Antigencharakter des Alpha-K.

378 Kuhhornstoß, eine erfolgreiche Ka-

taraktextraktion durch - 55 Kupfersplitter, Entfernung fein-

ster - mit der Löffelpinzette aus

dem Augeninnern Ill

$\mathrm{L}$

Lampe, einíache - zur Ophthal-

moskopie 115 Leukome, angeborene - der Horn-

haut 187 Lichtsinn, Verfahren zur zahlen-

mäßigen Bewertung des - 69 I Lidhämangiom 117 Ligamentum canthi interni,

Lockerung des - und Blepharo-

phimosis medialis senilis 118 I Lindausche Krankheit 399 Linse, Aplasie und Hypoplasie der

- $\quad$ 386Linsendislokationen 398Linse nektopie, Wanderlinse als

Folgezustand einer kongenitalen

- $\quad 160$

Linsenkapsel, Verhalten der -

nach Verletzung 378 Linsenluxation, habituelle und

spontane 20 - totale - bei Arachnodaktylie 388 Linsenvorderkapsel, histologi-

scher Befund der Feuerlamelle der

- $\quad$ 387Lipoidgranulomatose 120Lipoidosen, Chemismus der Zellen

und Gewebe in Beziehung $\mathrm{zu}-401$

Löffelpinzette, Entfernung fein-ster Kupfersplitter aus dem Augeninnern mit der - Ill

Lymphadenose, doppelseitigealeu-kämische - 187

$\mathrm{M}$

Magenkrebs oder Bleivergiftung

als Ursache einer toxischen Am-

blyopie 108 Marfanscher Symptomenkom-

plex mit totaler Linsenluxation

388 Marmorknochenkrankheit,

Atrophia nervi optici bei - 97 Masern, Enzephalitis nach - 384 Melanome des Fundus 389

Membrana epipapillaris 381 Meningiome suprasellare (intra-

kranielle) und orbitale - 124 Meningitis basilaris infolge von

Sehnerventuberkulose 195 Migräne und Netzhautablösung 191

Sachregister zu Band 75.405

Mikroorganismen, Wirkung äthe-rischer Öle auf augenpathogene -

279

- $\quad$ Wirkung der Grenzstrahlen auf- 280

Minderdrucktrauma am Auget88 Molluskum-Conj unctivitis 117 Myiasis 193

My o pie, hochgradige - in den $\mathbf{m}$ ersten Lebensjahren 189

- $\quad$ Einfluß vielfacher druckentlasten-der Operationen bei Hydrophthal-mus congenitus und gleichzeitigeEntstehung von ho her -17

$\mathrm{N}$

Naevus vasculosus der Conjunctiva und Episklera 136 Nervenfasern, markhaltige 106 Nervus optieus, posttraumatische Prominenz des - 108

Meningitis basilaris infolge von Tuberkulose des - 195

Tuberkulide des - als Kompli-kation von Hautsarkoiden 400 
- atrophie, hereditäre 108

- bei Marmorknochenkrankheit

97 Netzhaut s. Retina Netzhautablösung s. Ablatio

retinae Neuritis optica, verursacht durch

Erkrankung der Keilbeinhöhle

383 Neuritis retrobulbaris, Atiologie

der - 264 Nystagmus nach schwerer Schädel-

verletzung 107

der Bergleute 281

optokinetischer - im Dienste der Praxis 146, 185

0

Oberkiefer, Osteomyelitis des - beim Säugling 240, 280

Öle, Wirkung ätherischer - auf augenpathogene Mikroorganismen 279

Ophthalmia sympathica, Zell-studien bei - 382

Ophthalmomyiasis externa 193

Ophthalmoplegia externa et interna, traumatische 374

Orbitaltumor, Exophthalmus bei benignem - 197

- Veränderungen am Bulbus durchDruck von - 264

Osteomyelitis desOberkiefers beim Säugling 240, 280

$\mathrm{P}$

Pannus trachomatosus, medika-mentöse und operative Behand-lung des - 177

- Denigsche Operation gegen 194

Pantokain 125

Papillen, ungleich große -100

Perimetrie, Fixationsvorrichtung für - 105

Photoskop, Skiaskopie mit dem - 88, 102

Pigmentdegeneration der Netzhaut mit progressiver Aderhaut-atrophie 279

Pigmentstreifenerkrankung der Netzhaut 199

Plexus chorioideus, Kalkablage-rungen im - bei Retinitis pig-mentosa 164

PseudoisochromatischeTafeln, Farbenanalyse der - 196

Pseudoxanthoma elasticum der Haut und Pigmentstreifenerkrankung der Netzhaut 199

Psychiatrisch-neurologische Begutachtung in der Lebens-versicherungsmedizin 204

Pupillenfasern, besondere - für die indirekte Reaktion 22

$\mathrm{R}$

Refraktionsverminderung durch

Trauma 394 Retina, Messungen an den Arterien

der - bei beginnender Arterio-

sklerose 381

Stoffwechsel der -380

Vitamin A in der -382

Angiopathia retinae traumatica ad maculam 118

Pigmentstreifenerkrankung der - 199

Pigmentdegeneration der - mit progressiver Aderhautatrophie 279

Schaffung flächenhafter Verlö-tungen der - mit der Chorioidea durch Endothermie 207

Angio-Retikulo-Xanthom der -

399 Retinitis albuminurica 120 Retinitis pigmentosa, Kalkab- 
lagerungen im Plexus chorioideus

bei - 164 Retinitis pseudoalbuminurica

(stellata) nach Enzephalitis no Röntgenologie des Foramen op-

ticum 27 Röntgenuntersuchung, Wert der

- bei Erkrankungen der Augen-

höhle 243

406 Sachregister

S Sarkoide der Haut, kompliziert mit

Tuberkuliden des Sehnerven 400 Sarkom der Iris 257 Schädelverletzung, Nystagmus

nach - 107 Schilddrüsentumor, doppelseitige

Trochlearisparese als einzige Mo-

tilitätsstörung bei - 58 Schularbeit, Wirkung der - auf

die Augenfunktionen der Schüler

197 Schüllersche Krankheit 120 Schutzbrille 100 Schutzvorrichtungen, neue 204

Schwangerschaftsunterbre-

chung wegen Augenleiden 190 Schwangerschaftsveränderung

der Hypophyse als Ursache bitem-

poraler Hemianopsie 343 Schwefelwasserstofferkran-

kung der Augen 203 Sehnerv s. Nervus opticus Skiaskopie mit dem Photoskop 88,

102 - Zylinder-S. 195 Skier a, subkonjunktivale Zerrei-

Bung der - durch stumpfe Ge-

walteinwirkung 106 Skorbut, Exophthalmus bei Säug-

lings- 373 Soemmeringscher Kristall-

$\mathrm{w} \mathrm{u} 1 \mathrm{~s} \mathrm{t}$ in myopischen nach Fukala

operierten Augen 189 Spaltlampenmikroskopie, Lehr-

buch und Atlas der - 205 (Bb.) Splitterfreies Glas, Brillen aus -

105 Starkstromschädigung der Augen 193 Staubschädigungen 126 Stauungspapille bei

intrakrani-

eller Drucksteigerung 385 - und Blindheit bei Guernsey-

Stierkälbern 383 Stereoskopischer Atlas der äu-

Beren Erkrankungen des Auges

205 (Bb.) Strabismus 359

$\mathrm{T}$

Teilmattierte Gläser 96 Tetanie, Katarakt bei postopera-

tiver - 374 Thyreoidea, Beziehungen zwischen

Hypophyse und - 137 Tonometerkurven, dieallgemeine

Richtung normaler - 396 Trachom, die Lehre vom - einst

und jetzt 11

zu Band 75.

Trägerrahmen für Brillengestell

100 Tränendrüsen, Tumoren der -114 Tränenpunkt, Verlegung des -

eine Operation gegen Tränenfluß

117

- $\quad$ und Tränenkanälchen, konge-nitales Fehlen der - 374

Tränenröhrchen, Operationen an

den - 237 Tränenwege, röntgenologischeDar- 
stellung der - 187 Transplantation der Cornea 194 Trochlearisparese, doppelseitige - $\quad$ als einzige Motilitätsstörungbei Schilddrüsentumor 58

Tuberkulide des Sehnerven als Komplikation von Hautsarkoiden 400

Tuberkulose des Auges 392

Autoserumtherapie gegen -118

des Sehnerven, Meningitis basi-laris infolge von -195

U Überdrucktrauma am Auge 188 Ulcus serpens, Klinik und Behand-

lung des - 304 Ultraviolettes Licht, Behand-

lung von Augenleiden mit - 379 Unfallkunde 125, 190, 203, 281

$\mathrm{V}$

Verätzungen, Verlauf schwerster

- $\quad$ in der ersten 8 Tagen 282Vergiftungen 203Verknöcherung des Bulbus, Röntgenbefund bei - no Versicherungskunde 125, 203,

281 Vitamin A in der Netzhaut 382

W

Wanderlinseals Folgezustand einer kongenitalen Linsenektopie 160

Wilsonsche Krankheit mit Kay-ser-Fleischerschem Ring 103

$\mathrm{X}$

Xanthom, Angio-Retikulo-X. der Netzhaut 399

Z

Zentralnervensystem, traumatischer Diabetes nach Verletzung des - 128 Ziliarnervenschleifen, intraskle-

r. rale 376 Zweistärkengläser, drehbare 175 Zyklodialyse, Resultate der bei Glaucoma simplex 375 Zylinderskiaskopie 195 